\title{
Effect of Buyang Huanwu Decoction Combined with Acupuncture on Sequelae of Cerebral Stroke
}

\author{
Jian Guo, Deqi Ren, Xuehui Chang \\ Henan Provincial Hospital of Traditional Chinese Medicine, Zhengzhou, Henan \\ Henan Provincial Hospital of Traditional Chinese Medicine, Zhengzhou, Henan \\ Henan University of Chinese Medicine, Zhengzhou, Henan
}

\begin{abstract}
Objective: To explore the clinical effect of the combined use of Buyang Huanwu Decoction and acupuncture in the treatment of patients with sequelae of stroke. Methods: The research work was carried out in our hospital. The study started from September 2018 to September 2019. A total of 100 patients were selected and randomly grouped into experimental group and control group, both groups were given Buyang Huanwu Decoction for intervention, the experimental group were added acupuncture treatment methods on this basis to explore the clinical effects of different intervention methods. Results: There were 4 patients with ineffective treatment in the experimental group, the effective rate was $92.00 \%$, and the effective rate in the control group was $74.00 \%$. The effective rate of the experimental group was higher, and the data was significantly different $(\mathrm{P}<0.05)$. After treatment, the FMA, NIHSS score and Barthel index of the patients in the experimental group were $(32.66 \pm 6.51),(7.11 \pm 3.54)$ and $(90.34 \pm 9.87)$ respectively. The FMA score and Barthel index were significantly higher than the control group, and the NIHSS score was lower than the control group, data differences are significant $(\mathrm{P}<0.05)$. At the same time, before treatment, there was no significant difference in the scores of TCM syndromes. After treatment, the patients in the experimental group had less hemiplegia, skewed eyes and mouth, eccentric sensation abnormality and consciousness coma scores than that of the control group. The data difference is quite large $(\mathrm{P}<0.05)$, the experimental group had better effect. Conclusion: The combined treatment of Buyang Huanwu Decoction and acupuncture in the treatment of patients with sequelae of stroke has a significant clinical effect, which can improve the quality of life of patients, improve the nerve function of patients, and have a positive effect on clinical development.
\end{abstract}

\section{1 introduction}

In clinical development, stroke symptoms are relatively common, mainly cerebrovascular diseases, and the morbidity of patients is relatively high, and it is often accompanied by various complications, resulting in high clinical mortality of the disease, which has serious threat to life and health of patients. For most stroke patients, after surviving, they are often accompanied by a variety of different levels of speech dysfunction, limb movement disorders, and cognitive impairments. The patients can not take care of themselves for most of their lives, causing serious adverse effects on the patients' psychology. From the perspective of clinical development in recent years, when patients are given acupuncture combined with traditional Chinese medicine, the prognostic effect of patients can be improved and their ability to take care of themselves can be improved [1]. This research is based on this, applying this method, carrying out relevant research in our hospital, and exploring its clinical application efficiency.

\section{General information and research methods of patients}

\subsection{General information analysis}

The research work was started in our hospital. The study started from September 2018 to September 2019. A total of 100 patients were selected and randomly grouped. They were divided into the experimental group and the control group. Both groups were given Buyang Huanwu Decoction, the experimental group were added acupuncture treatment on this basis, the number of patients in each group was 50, all patients have sequelae of stroke. Among the patients, there were 55 males and 45 females. The age of the patients ranged from 43 to 76 years, the corresponding average age was $(62.34 \pm 3.15)$ years, the course of disease was 1-4 years, and the average course was $(1.34 \pm 0.32)$ year. There was no significant difference in general data between the two groups of patients, and comparison between the groups was possible $(\mathrm{P}>0.05)$. 


\subsection{Research methods}

In this study, patients in the control group were given Buyang Huanwu Decoction with addition and subtraction treatment. The specific prescriptions were: $60 \mathrm{~g}$ astragalus, $20 \mathrm{~g}$ dilong, $10 \mathrm{~g}$ safflower, 20g Chuanqiong, 20g red peony, $10 \mathrm{~g}$ peach kernel and $20 \mathrm{~g}$ angelica tail. For patients with skewed eyes and mouth, patients need to be given white aconite and scorpion; For patients with hemiplegia symptoms, pangolins, leeches and mulberries need to be added to them; If the patient is accompanied by dizziness and headache, then Gastrodia and Uncaria paralysis and weakness, ginseng and Guizhi need to be added. During the administration, all medicines need to be soaked in warm water for 30 minutes, followed by two decoctions to concentrate the drug solution to $400 \mathrm{ml}$, which are divided into two doses, one for each morning and evening, one dose per day.

The patients in the experimental group used the treatment method of Buyang Huanwu Decoction combined with acupuncture and moxibustion. The prescription and dosage of Buyang Huanwu Decoction were the same as those in the control group. For acupuncture treatment, the main points include Sanyinjiao, Weizhong, Neiguan, Jiquan, Chize and Shuigou. For patients with skewed eyes and mouth, it is also necessary to increase the intervention of acupoints such as Zanzhu, Renzhong, Baihui, Xiache, and Dicang; while for patients with upper limb failure, it is necessary to increase the acupoints intervention of Shousanli, Neiguan, Quchi, and Hegu; if the patient is complicated with upper limb failure, it is necessary to increase Xuehai, Huantiao, Taixi, Sanyinjiao and Yaoyangguan acupoints. In the actual operation process, it is necessary to apply the $25 \mathrm{~mm}$ needle, and to intervene with the method of supplementing the deficiency, lifting and inserting and rolling, and to retain the needle for 30 minutes, intervene once a day, after giving the patient 5 days of intervention, stop for two days, then repeat the treatment, patients in both groups had received continuous treatment for 3 months. also need to be added; For patients with upper limb

\subsection{Observation indicators}

Effective rate of treatment mainly includes marked effective, effective and ineffective. Among them, marked effective patients mainly showed that clinical symptoms were improved, various limb functions and nerve functions were restored, and patients can achieve selfcare; Effective patients showed that the clinical symptoms of the patients can be effectively improved, the limb motor function and language function can be restored, and simple daily life can be carried out; Ineffective patients indicated that the patients had not improved any vital signs and limb functions after receiving treatment. The effective rate of this study is the total probability excluding inefficiency.

The neurological impairment scoring standard was applied, with a score of $0-45$, mild at $0-15$, moderate at 16-30, and severe at 31-45. According to the evaluation of patients' daily life quality and limb motor function, the Barthel index scale and FMA score were mainly used.

The scores of TCM syndromes mainly include abnormal body sensation, hemiplegia, crooked eyes and mouth, and dizziness. The maximum score for each item is 6 points. The higher the score, the more obvious the patient's syndrome.

\subsection{Statistical methods}

Using the statistical software SPSS20.0 as a tool, performing statistical analysis on the data presented in this study, verifying the $t$ value of the comparison result of the measurement data $( \pm \mathrm{s})$, and verifying the 2 value for the comparison result of the count data (n, \%), when the result shows $\mathrm{P}<0.05$, it means that the difference between the groups has statistical analysis value [2].

\section{Results}

\subsection{Treatment effective rate}

There were 4 patients who were ineffective in the experimental group, the effective rate was $92.00 \%$, and the effective rate in the control group was $74.00 \%$. The effective rate of the experimental group was higher, and the data was significantly different $(\mathrm{P}<0.05)$.

Table 1 Analysis of the treatment effective rate of the two groups of patients [n(\%)]

\begin{tabular}{ccccc}
\hline Group & Marked effective & Effective & Ineffective & Effective rate \\
\hline $\begin{array}{c}\text { Experimental } \\
\text { group }(\mathbf{n}=\mathbf{5 0})\end{array}$ & $34(68.00)$ & $12(24.00)$ & $4(8.00)$ & $46(92.00)$ \\
$\begin{array}{c}\text { Control group(n=50) } \\
\mathrm{X}^{2}\end{array}$ & $17(34.00)$ & $20(40.00)$ & $13(26.00)$ & $37(74.00)$ \\
$\mathrm{P}$ & & & & 5.048 \\
\end{tabular}

lower than the control Group, the effect is better, and the

\subsection{Comparison of indicator scores}

data differences are significant $(\mathrm{P}<0.05)$.

After treatment, the FMA, NIHSS score and Barthel index of the patients in the experimental group were (32.66 \pm 6.51$),(7.11 \pm 3.54)$ and $(90.34 \pm 9.87)$ respectively. The FMA score and Barthel index were significantly higher than the control group, and the NIHSS score was 
Table 2 FMA, NIHSS score and Barthel index score $( \pm s)$ of the two groups of patients after treatment

\begin{tabular}{cccc}
\hline Group & FMA Score & NIHSS Score & Barthel Index \\
\hline Experimental group(n=50) & $32.66 \pm 6.51$ & $7.11 \pm 3.54$ & $90.34 \pm 9.87$ \\
& $25.04 \pm 5.32$ & $12.35 \pm 4.35$ & $73.65 \pm 8.55$ \\
$\mathrm{t}$ & 11.325 & 5.648 & 23.640 \\
Control group $(\mathbf{n}=\mathbf{5 0}) \mathrm{P}$ & $<0.05$ & $<0.05$ & $<0.05$ \\
\hline
\end{tabular}

abnormality and consciousness coma scores of the

\subsection{Comparison of TCM Syndrome Points}

Before treatment, there was no significant difference in the scores of TCM syndromes. After treatment, the hemiplegia, skewed eyes and mouth, eccentric sensation

Table 3 Comparison of TCM Syndrome Points of Two Groups of Patients after Treatment $( \pm \mathrm{s})$

\begin{tabular}{ccccc}
\hline Group & hemiplegia & $\begin{array}{c}\text { skewed eyes and } \\
\text { mouth }\end{array}$ & $\begin{array}{c}\text { eccentric sensation } \\
\text { abnormality }\end{array}$ & consciousness coma \\
\hline $\begin{array}{c}\text { Experimental } \\
\text { group(n=50) }\end{array}$ & $1.55 \pm 0.32$ & $1.54 \pm 0.65$ & $1.94 \pm 0.33$ & $1.34 \pm 0.24$ \\
Control group(n=50) & $2.41 \pm 0.55$ & $2.74 \pm 0.59$ & $2.11 \pm 0.48$ & $2.07 \pm 0.35$ \\
$\mathrm{t}$ & 4.035 & 6.220 & 5.947 & 6.644 \\
$\mathrm{P}$ & $<0.05$ & $<0.05$ & $<0.05$ & $<0.05$
\end{tabular}

\section{Discussion}

Judging from the current development situation, the aging problem in China has gradually deepened, leading to the gradual increase in the incidence of stroke. In clinical development, stroke symptoms are relatively common, which are mainly cerebrovascular diseases, and the incidence of patients is relatively high, and many with various complications, the clinical mortality of the disease is high, which poses a serious threat to the life and health of patients [3]. For the majority of stroke patients, after surviving, they are often accompanied by a variety of different levels of speech dysfunction, limb movement disorders, and cognitive impairment. The patients can not take care of themselves, such as poststroke spastic paralysis, etc., which greatly affects the quality of life of patients and even cause serious adverse effects on the patient's psychology, which is not conducive to the patient's recovery. However, in the development of clinical treatment of cerebral sequelae of stroke, certain principles need to be followed, which mainly are: to improve the patient's muscle tone, alleviate the degree of edema of the patient's limbs, and achieve the improvement of the patient's local blood supply, but in actual development, patients need to be given modern rehabilitation therapy, the corresponding effect is easily affected by many factors, which will reduce clinical efficacy [4]. In this process, the use of traditional Chinese medicine treatment methods to intervene in patients with sequelae of stroke has significant effects and unique application advantages.

From the perspective of motherland medicine, the pathogenesis of stroke sequelae mainly manifests as qi deficiency, blood stasis, and poor blood circulation. In the process of giving patients treatment, it is necessary to apply the principles of promoting blood circulation and removing blood stasis, supplementing qi and blood, and promoting dampness and removing phlegm [5]. The Buyang Huanwu Decoction, which contains astragalus, has the effects of removing blood stasis, and nourishing vital energy, while the application of angelica, chuanqiong, red peony, and peach kernel can achieve the benefits of nourishing qi and blood, promoting blood circulation and removing blood stasis, as for Dilong, its corresponding effects of activating meridians and channels are obvious, and when a variety of drugs are used in combination, the effect of removing blood stasis, clearing collaterals, strengthening vital energy and removing pathogenic factors can be achieved [6]. From the perspective of modern pharmacological research, the application of Buyang Huanwu Decoction has a relatively significant clinical effect, which can improve the permeability of capillaries, promote the scavenging effect of free radicals, and achieve effective platelet aggregation inhibition, while achieving improvement in blood circulation, promote the increase of patients' vascular endothelial function, and achieve effective recovery of patients' brain cell function [7]. Acupuncture treatment method is the main method of traditional Chinese medicine treatment. In the specific implementation process, by intervening and stimulating the acupuncture points, the function of the body's blood and viscera is improved, and the patient's self-care ability is improved. When the two methods are used in combination to achieve treatment of patients with stroke sequelae, the corresponding clinical effect is more significant [8]. 
The results of this study showed that there were 4 patients in the experimental group who were ineffective, with an effective rate of $92.00 \%$ and an effective rate of $74.00 \%$ in the control group. The effective rate of the experimental group was higher, and the data was significantly different $(\mathrm{P}<0.05)$. After treatment, the FMA, NIHSS score and Barthel index of the patients in the experimental group were $(32.66 \pm 6.51),(7.11 \pm 3.54)$ and $(90.34 \pm 9.87)$ respectively. The FMA score and Barthel index were significantly higher than the control group, and the NIHSS score was lower than the control group, the data differences are significant $(\mathrm{P}<0.05)$. At the same time, before treatment, there was no significant difference in the scores of TCM syndromes. After treatment, the patients in the experimental group had less hemiplegia, skewed eyes and mouth, abnormal body sensation and consciousness coma scores than the control group. The data difference is quite large $(\mathrm{P}<0.05)$, the experimental group had better effect.

In summary, the combination of Buyang Huanwu Decoction and acupuncture in the treatment of patients with sequelae of stroke has a remarkable corresponding clinical effect. It can promote the improvement of patients' quality of life, improve patients' nerve function, and have a positive effect on clinical development.

\section{Acknowledgement}

In the process of writing the thesis, my teacher gave me great help and encouragement. Under his guidance, my professional knowledge level has been significantly improved and expanded. Here, I would like to thank my teacher sincerely

\section{References:}

1. Wu Xiuzhen, "Effect of Buyang Huanwu Decoction combined with acupuncture on 64 cases of sequelae of cerebral stroke[J]" in Clinical Research of Traditional Chinese Medicine, 2015,7(06):50-51.

2. Huang Xiaojun, "Observation of Curative Effect of Buyang Huanwu Decoction Combined with Acupuncture on Cerebral Apoplexy[J]" in Popular Health (Academic Edition),2015,9(09):34-35.

3. Liu Yuecheng, "Wang Sufang, Gao Mengge. Observation of the therapeutic effect of Buyang Huanwu Decoction combined with acupuncture on sequelae of cerebral stroke[J]" in Electronic Journal of Clinical Medicine and Literature,2015,2(17):3477.

4. Li Suwen, "Observation on the Curative Effect of 39 Cases of Cerebral Apoplexy Treated with Buyang Huanwu Decoction Combined with Acupuncture and Moxibustion[J]" in Clinical Research of Chinese Medicine, 2016,8(10):32-33.

5. Liang Zengkun, Zhang Limin, Hu Xun, "Clinical Observation of Buyang Huanwu Decoction Combined with Acupuncture and Moxibustion for Treatment of Sequelae of Cerebral Stroke[J]" in Guangming Traditional Chinese Medicine,2018,33(04):459-460.
6. Yang Xiaofeng, "Observation on the Curative Effect of Buyang Huanwu Decoction Combined with Acupuncture on Cerebral Apoplexy[J]" in Shanghai Medical, 2018,39(17):37-39.

7. Qin Chunjie, "Clinical Observation of Buyang Huanwu Decoction Combined with Acupuncture and Moxibustion for the Treatment of Sequelae of Stroke[J]" in Chinese Urban and Rural Enterprise Health, 2017,32(05):115-116.

8. Liu Qiong, "Clinical effect of Buyang Huanwu Decoction + acupuncture on patients with sequelae of cerebral stroke[J]" in World Latest Medical Information Digest, 2017,17(50):89+92. 